\title{
Field emission from laser-processed niobium (110) single crystals
}

\author{
V. Porshyn, ${ }^{*}$ P. Serbun, and D. Lützenkirchen-Hecht \\ Faculty of Mathematics and Natural Sciences-Physics, University of Wuppertal, \\ 42119 Wuppertal, Germany
}

(Received 20 November 2018; published 11 February 2019)

\begin{abstract}
Niobium is a technologically important material, which is typically used for superconducting radiofrequency applications. Superconductive cavities made of niobium require contamination-free and smooth surfaces to ensure the best performance of a particle accelerator. Interior surfaces of niobium cavities are usually obtained by centrifugal barrel polishing, buffered chemical polishing, and electropolishing or a combination of these methods. However, a standard inspection of the inner cavity surface after the treatment still shows the presence of sharp features limiting cavity performance. Laser polishing is a potential alternative or can be used as an additional final step for a more efficient surface treatment toward higher electric field gradients. In the present study, a chemically polished (110)-oriented single crystal niobium surface was processed with a focused pulsed laser with a pulse duration of $3.5 \mathrm{~ns}$ and a repetition rate of $10 \mathrm{~Hz}$. The laser fluence and the number of pulses were varied in the range from 0.68 up to $4.27 \mathrm{~J} / \mathrm{cm}^{2}$ and from 20 up to 200 , respectively. The magnitude of laser-induced surface structures and boiling traces was systematically studied by means of scanning electron microscopy, atomic force microscopy, and optical profilometry. Finally, the local field emission behavior was investigated and correlated with the observed surface modifications. Typical current-field characteristics and a field enhancement statistic of laser-processed areas are presented in the study. The surface processing with a rather low laser fluence of $0.68 \mathrm{~J} / \mathrm{cm}^{2}$ yielded high onset electric fields of $650-940 \mathrm{MV} / \mathrm{m}$ with field enhancement factors below 10 . The processing with a higher laser fluence and/or a higher number of pulses resulted in boiledlike structures emitting at $70-190 \mathrm{MV} / \mathrm{m}$.
\end{abstract}

DOI: 10.1103/PhysRevAccelBeams.22.023101

\section{INTRODUCTION}

The melting of thin surface layers by intense laser illumination is an important phenomenon, which can be used for surface polishing to a mirrorlike finish. The successful application of laser polishing has already been demonstrated for a number of metals, e.g., aluminum, titanium, and steel alloys, as well as for fused silica [1-6]. An efficient melting with a laser beam can be achieved, in particular, with nanosecond pulsed radiation [7]. Selective and force-free processing without any impact of polishing agents and wastes makes this method very attractive also for industrial applications, avoiding any contaminations of the surfaces with abrasives or chemicals. A reduction of the surface roughness from initial values of several tens of microns down to a submicron range was found to be

\footnotetext{
*To whom all correspondence should be addressed. porshyn@uni-wuppertal.de

Published by the American Physical Society under the terms of the Creative Commons Attribution 4.0 International license. Further distribution of this work must maintain attribution to the author(s) and the published article's title, journal citation, and DOI.
}

achievable for proper process parameters [8-10], i.e. laser intensity, pulse duration, repetition rate, and total number of pulses. For large area polishing, the feed rate and the spatial overlap of individual laser pulses are also important. In our study, the systematically varied key parameters were the laser fluence $(F)$ and the number of pulses per spot $(N)$. Finally, the local field emission behavior of laser-processed areas was measured and correlated with the parameters of the illumination and the created surface modifications.

Our target materials were flat, (110)-oriented highpurity $\mathrm{Nb}$ single crystals with a residual-resistance ratio of above 250, which are of particular interest for particle accelerators, where niobium superconducting radiofrequency (SRF) cavities are widely used. Since the superconducting rf currents flow only in a thin surface layer about $50 \mathrm{~nm}$ thickness, the preparation of smooth surfaces is of key importance for the performance of the cavity. Contaminations and surface defects may lead to parasitic field electron emission and, as a consequence, limit achievable acceleration gradients. Furthermore, the mechanical forming of the cavities gives rise to a surface damage layer of about $100 \mu \mathrm{m}$ thickness which has to be removed prior to the cavity operation at high field gradients. Applying conventional polishing methods like buffered 
chemical polishing (BCP) and electropolishing (EP), $\mathrm{Nb}$ cavities allow one to reach acceleration gradients of up to 45.5 MV/m [11,12]. The achievable acceleration gradient is limited due to numerous sharp surface structures and particulates, which exhibit high field enhancement factors, leading to a strongly enhanced parasitic field emission or/ and a magnetic field enhancement [13-15]. Previous studies of similar samples showed that it is not sufficient just to remove the oxide layer by a heat treatment to limit the number of parasitic field emitters [16]. Overall surface damage should be repaired, and a high surface purity should be achieved to improve the quality of SRF cavities. This goal is feasible by remelting a surface layer with intense nanosecond-laser pulses. Also, the traditional polishing techniques involve some other issues, in particular, the utilization of significant amounts of hazardous and corrosive liquids such as concentrated hydrofluoric (HF), nitric $\left(\mathrm{HNO}_{3}\right)$, sulfuric $\left(\mathrm{H}_{2} \mathrm{SO}_{4}\right)$, and phosphoric $\left(\mathrm{H}_{3} \mathrm{PO}_{4}\right)$ acids [17], which can be entirely avoided using laser polishing. Thus, laser polishing represents a promising alternative for established polishing procedures or at least an additional final preparation step, which can provide sufficiently smooth surfaces of $\mathrm{Nb}$, as was shown in earlier studies [18]. Numerous sharp features of the BCP-treated polycrystalline $\mathrm{Nb}$ surfaces that may lead to parasitic field emission and finally to a breakdown of superconductivity could be successfully rounded. In additional, the purity of the upper surface layer was improved. However, the grain boundaries remained unchanged. In the present study, we have followed the processing with a nanosecond-pulsed laser by means of electron microscopy (SEM), optical profilometry, and atomic force microscopy (AFM), and a subsequent analysis of the processed specimen surfaces with field emission scanning microscopy (FESM) was conducted.

\section{EXPERIMENTAL}

Identical niobium crystals were treated elsewhere employing common techniques which are usually used for the polishing of superconducting cavities as described in Ref. [19]. Surface layers of 140 and $40 \mu \mathrm{m}$ were removed by $\mathrm{BCP}$ and a subsequent EP, respectively, followed by a high-pressure rinsing with ultrapure water as the final cleaning step, providing a mirrorlike surface finally. The preparation and installation of the samples in the vacuum chambers was done under cleanroom conditions (ISO 4).

The analysis of the $\mathrm{Nb}$ single crystal surfaces was done with a high-resolution scanning electron microscope SEM (JEOL JSM-6510 system with a resolution of ca. $3 \mathrm{~nm}$ ), an optical profilometer (OP, FRT MicroProf system with a lateral resolution of ca. $2 \mu \mathrm{m}$ and a vertical resolution of ca. $3 \mathrm{~nm}$ ), and an atomic force microscope (AFM, FRT MicroProf system with a lateral resolution of $3 \mathrm{~nm}$ and a vertical resolution of $1 \mathrm{~nm}$ ). Typical values for the root-mean square roughness $\left(R_{q}\right)$ of large areas of ca. $10 \times 10 \mathrm{~mm}^{2}$ amount to be around $100 \mathrm{~nm}$ as measured with using the OP. Local measurements of small areas of ca. $0.1 \times 0.1 \mathrm{~mm}^{2}$ with AFM resulted in values of 5-14 nm. Typical surface defects remaining after the conventional processing were scratches with a depth of some tens of nanometers. X-ray diffraction with the $X^{\prime}$ Pert Pro multipurpose diffractometer system (Philips/Panalytical) using $\mathrm{Cu} \mathrm{K}-\alpha$ radiation proved the (110) orientation of the crystals.

The circular $\mathrm{Nb}$ (110) single crystal samples with a diameter of $25 \mathrm{~mm}$ and a thickness of ca. $3 \mathrm{~mm}$ were placed on an electrically isolated manipulation rod inside of a vacuum chamber with a base pressure of ca. $4 \times 10^{-6} \mathrm{~Pa}$ and illuminated by a pulsed tunable $\mathrm{Nd}$ :YAG laser (EKSPLA NT342A-SH) as shown schematically in Fig. S1 [20]. All measurements were performed at room temperature. The third harmonic of the laser with a wavelength $\lambda=355 \mathrm{~nm}$ and a pulse energy of up to $3 \mathrm{~mJ}$ was used in the experiments; this wavelength can be well absorbed by the niobium material. The repetition rate was $10 \mathrm{~Hz}$, and the duration of a single laser pulse was 3.5 ns. Furthermore, the Gaussian laser beam was transmitted through the exit slit with a diameter of $4 \mathrm{~mm}$ and then focused by a fused silica lens with a focal length of $75 \mathrm{~mm}$, which could be precisely moved in any direction relative to a sample. Thus, the spot size, the spot shape, and the position of the spot on the sample were defined by moving the lens only, in general, with micron-level precision. The adjusted spot size was directly measured with a long-distance microscope (BW1008-500X). The corresponding minimal achievable circular spot size $\left(\varnothing_{\min }\right)$ at normal incidence was found to be around $210 \mu \mathrm{m}$. The pulsed output energy $(W)$ was determined separately by a pyroelectric sensor (Ophir Optonics PE25BF), and the resulting incident laser fluence $(F)$ was calculated using $F=0.86 \mathrm{~W} /\left[\pi\left(\emptyset_{\min } / 2\right)^{2}\right]$, taking into account the transmission of the lens and the vacuum window with a value of $86 \%$ at a wavelength of $355 \mathrm{~nm}$ [21]. The total relative error for $F$ was estimated to be around 5\% due to fluctuations of $W$ and the fact that the shape of the spot became slightly elliptical for increasing incident angles. More details about the setup are given in the Supplemental Material [20].

Inside of the chamber, the sample holder on the rod was electrically separated from the ground and connected to a feedthrough with $50 \Omega$ impedance. Thus, the charge of the sample and the time-resolved voltage signals induced by single and multiple laser pulses could be directly measured by an electrometer and an oscilloscope. These additional electrical measurements were used to follow the laser processing in situ. A typical time-resolved sample voltage response is presented in Fig. S2 [20]. Future measurements are planned to study the time-dependent evolution of the sample voltage during pulsed laser illumination in more detail.

Local field emission studies were carried out employing FESM under a base pressure of ca. $10^{-8} \mathrm{~Pa}$ [22]. A positive 
voltage was applied to a tungsten anode with a truncated cone $16 \mu \mathrm{m}$ in diameter. The gap between the anode and the $\mathrm{Nb}$ sample was simultaneously observed from two directions (enclosed angle of ca. $90^{\circ}$ ) by means of two longdistance microscopes (Questar and K2 DistaMax) with an optical resolution of ca. $1 \mu \mathrm{m}$; see Fig. S3 [20]. A fine gap adjustment with a piezo-based linear motion drive was used for gaps smaller than $30 \mu \mathrm{m}$, giving a relative error of ca. $3 \%$ for the resulting electrical field $E$.

The measured $I_{\mathrm{FE}} E$-behavior was analyzed in terms of the Fowler-Nordheim (FN) theory:

$$
I_{\mathrm{FE}}=A \frac{S \beta^{2} E^{2}}{\phi t^{2}(y)} \exp \left(-B \frac{\phi^{3 / 2} v(y)}{\beta E}\right),
$$

with the field enhancement factor $\beta$, the emitting area $S$, and work function $\varphi$ [23]. The following parameters were used here: $\varphi=4.37 \mathrm{eV}$ [24], $v(y)=t(y)=1, A=$ $154 \times 10^{4} \mathrm{~A} \mathrm{eV} \mathrm{m}^{2} / \mathrm{MV}^{2}$, and $B=6830 \mathrm{MV} /\left(\mathrm{eV}^{2} \mathrm{~m}\right)$ for the determination of $\beta$ values from the Fowler-Nordheim plots $\left[\ln \left(I_{\mathrm{FE}} / E^{2}\right)\right.$ vs $\left.1 / E\right]$.

\section{RESULTS AND DISCUSSION}

\section{A. Morphology and topography of the laser-irradiated Nb surface}

In the following, the niobium samples were processed with the focused laser beam applying pulse trains with different numbers of pulses (up to 200) and a variation of the laser fluence $(0.68,1.40,2.07,2.89,3.53$, and $4.27 \mathrm{~J} / \mathrm{cm}^{2}$ ) at different lateral positions on the surface. Typical results for the observed changes of the surface after the laser treatment are presented in Fig. 1 for three selected laser fluences. It is expected that the solid material will melt if a sufficient number of laser pulses and a suited laser fluence are applied. From Fig. 1(a), it can be concluded that 120 pulses with $0.68 \mathrm{~J} / \mathrm{cm}^{2}$ already alter the surface substantially, and a further increase of the laser fluence and pulse number leads to highly degraded surfaces [Figs. 1(b) and 1(c)]. The shape of the generated craters develops from weakly pronounced wavelike structures [Fig. 1(a)], craters with additional small pinholes [Fig. 1(b)], and craters with numerous droplets and deep cavities [Fig. 1(c)] with increasing laser irradiation.

In general, the involved laser parameters implicate an optical absorption depth of ca. $10 \mathrm{~nm}$ [25]. In dependence on the laser fluence, the uppermost layers of the $\mathrm{Nb}$ are therefore heated to the melting point, and an evaporation of the target may occur. It is important to note that this is a fast process, which occurs during the first nanoseconds of the laser illumination [26]. A part of the beam is reflected first by the solid and then by the liquid. At a certain laser fluence, the vapor becomes dense enough to interact with the laser beam. At this point, the vapor becomes photoionized, and an additional shielding of the initial surface by the plasma takes place [27]. The melting front and the vapor expand in the target and the vacuum, respectively, after the laser pulse termination $[28,29]$. Finally, a recondensation of the vapor and a cooldown and resolidification of the target take place followed by the formation of self-organized surface structures [30].

According to the resulting morphological modifications of the $\mathrm{Nb}$ surface as detected by SEM, the first evidence for a melted surface was already detectable for a laser fluence of roughly $0.68 \mathrm{~J} / \mathrm{cm}^{2}$. This observation is close to the thermal modeling of the laser-induced heating [31-34], which predicts the melting threshold of $\mathrm{Nb}$ in the range of $1.1-1.9 \mathrm{~J} / \mathrm{cm}^{2}$ for single laser pulses, and a decrease of the ablation threshold is expected if high pulse repetition rates are applied [35]. Earlier studies of this pulse to pulse accumulation effect propose a power law for the dependency of the threshold $F_{\text {th }}$ on the number of pulses with $F_{\text {th }} \sim N^{-\gamma}$ and a positive parameter $\gamma \ll 1$ [36]. The accumulation of quenched-in extended defects in a near surface region of the illuminated material is suggested as the most possible reason for this phenomenon [37,38]. Furthermore, it has to be taken into account that the melting threshold strongly depends on the reflectivity of the illuminated surface and, thus, on the surface roughness as well [39]. The observation of melting processes for a
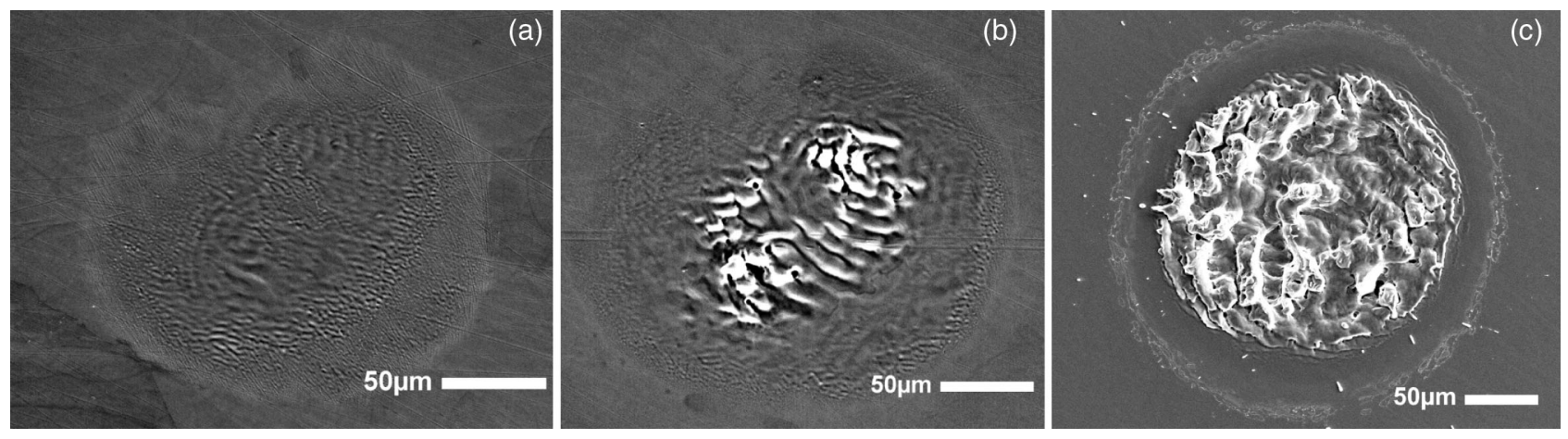

FIG. 1. SEM micrographs of the three characteristic types of craters created on the flat surface of Nb induced by a pulsed laser illumination: (a) $F=0.68 \mathrm{~J} / \mathrm{cm}^{2}, N=120$; (b) $F=1.40 \mathrm{~J} / \mathrm{cm}^{2}, N=100$; and (c) $F=2.89 \mathrm{~J} / \mathrm{cm}^{2}, N=140$. 
laser fluence of only $0.68 \mathrm{~J} / \mathrm{cm}^{2}$ applying more than 20 laser pulses [see Fig. 4(a)] thus appears well in accordance with literature data.

According to Fig. 1(b), smaller cavities occur in the $\mathrm{Nb}$ surface if the laser fluence is increased to $1.4 \mathrm{~J} / \mathrm{cm}^{2}$ (see also the inset in Fig. 2), that progressively develop when the fluence is further increased to values of more than $2.5 \mathrm{~J} / \mathrm{cm}^{2}$. The presence of small metallic spatters well outside the laser-illuminated area [see Fig. 1(c)] can be assumed as a clear indication for a boiling of the $\mathrm{Nb}$ surfaces - the material is spilled out and will condense on the unheated surface close to the illuminated area subsequently. Going one step further, one may even assume that the presence of the above-mentioned small pinholes is the first indication for boiling processes. We have thus performed a series of experiments in which the $\mathrm{Nb}$ samples were treated with pulse trains of different laser fluences and under variation of the number of applied laser pulses in order to find out the threshold for those traces. The results are compiled in Fig. 2. Based on the assumption that the pinholes that were observed in the ablated area resulted from a boiling of the liquefied metal, it can be suggested that, in fact, the melting threshold for $\mathrm{Nb}$ was significantly reduced from pulse to pulse by the repetition rate of $10 \mathrm{~Hz}$ in our experiment. Here it can be clearly seen that the threshold $F_{\text {boil }}$ for the occurrence of the mentioned features characteristic for a boiling of the liquefied $\mathrm{Nb}$ decreases significantly from about $3.5 \mathrm{~J} / \mathrm{cm}^{2}$ for 20 pulses to only $1.3 \mathrm{~J} / \mathrm{cm}^{2}$ for 80 pulses, while no boiling was observed for a fluence of $0.68 \mathrm{~J} / \mathrm{cm}^{2}$ and an accumulation of substantially more than 160 laser pulses. The pulse number was varied in a relatively small range compared to the findings in Ref. [36], so a rather linear decrease of $F_{\text {boil }}$ on $N$ in our case could be observed.

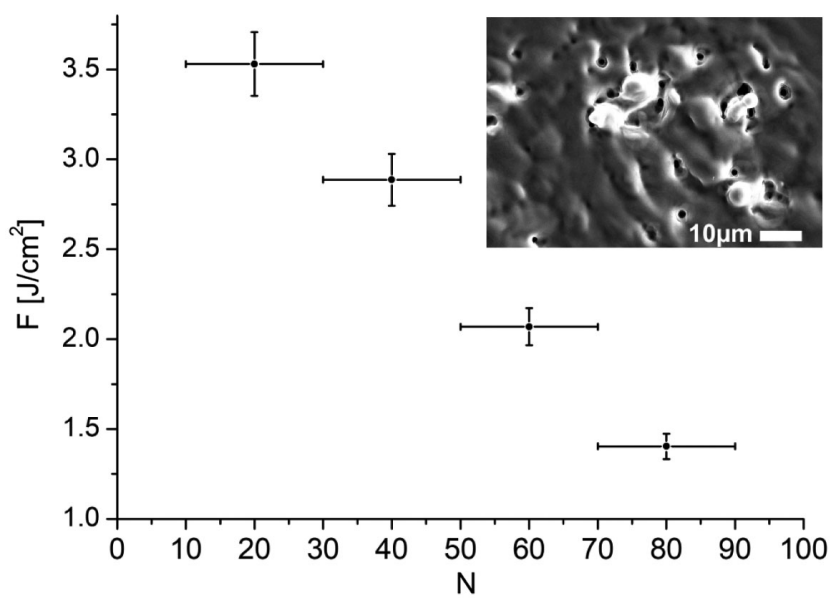

FIG. 2. The threshold for the occurrence of the boiling traces [see Fig. 1(b)] in dependence of the number of pulses, $N$, and laser fluence $F$. The inset shows a SEM micrograph of pinholes, which appear in the illuminated area for the corresponding values of $N$ and $F$; here $F=1.4 \mathrm{~J} / \mathrm{cm}^{2}$ and $N=120$.
The morphology of the smoothly ablated region [see Fig. 1(a)] basically corresponded to the Gaussian laser beam profile with the highest laser fluence in the central part of the spot (see Sec. II). The size of the melted area with a diameter of ca. $200 \mu \mathrm{m}$ was nearly equal to the nominal area of the focused laser spot. Wavelike structures with a characteristic lateral wavelength $\left(\lambda_{1}\right)$ systematically appeared at the borders of the melted area as can be seen in Fig. 3(a). This wavelength $\lambda_{1}$ was found to be comparable to the wavelength of the incident laser radiation and was also strongly dependent on the incidence angle of the laser beam. Thus, the observed phenomenon is related to the interference of the incident, linearly polarized laser radiation with electromagnetic waves scattered at the surfaces of the $\mathrm{Nb}[40,41]$. Their typical wave amplitudes were measured to be below $20 \mathrm{~nm}$ as determined by atomic force microscopy [see Fig. 4(a)].

A second type of circular periodic fringes with a different lateral wavelength $\lambda_{2}$ primarily appeared in the center of the melted region for longer pulse trains or an increasing laser
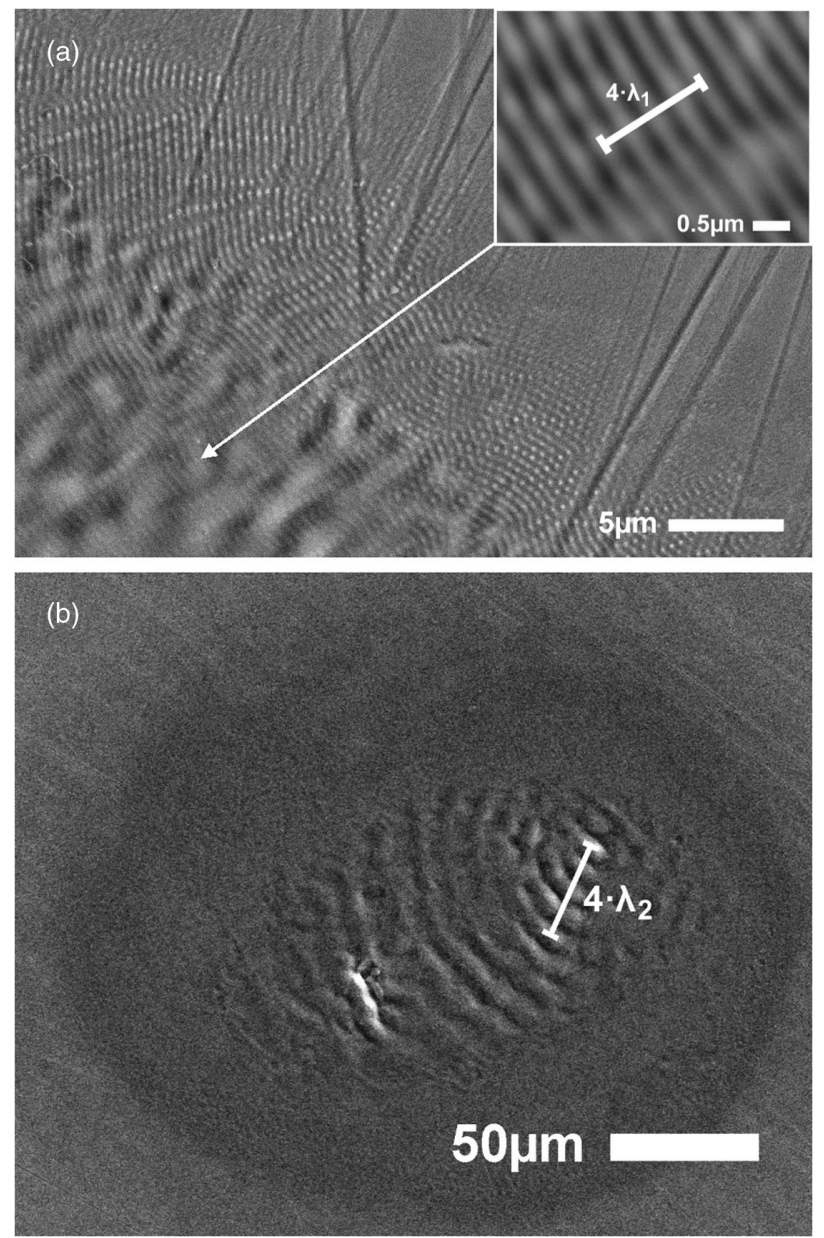

FIG. 3. SEM micrographs of the two characteristic types of waves propagating in the liquid phase with $\lambda_{1} \approx$ $400 \mathrm{~nm}$ (a) $\left(F=0.68 \mathrm{~J} / \mathrm{cm}^{2}, \quad N=180\right)$ and $\lambda_{2} \approx 8.3 \mu \mathrm{m}$ (b) $\left(F=1.4 \mathrm{~J} / \mathrm{cm}^{2}, N=80\right)$. 

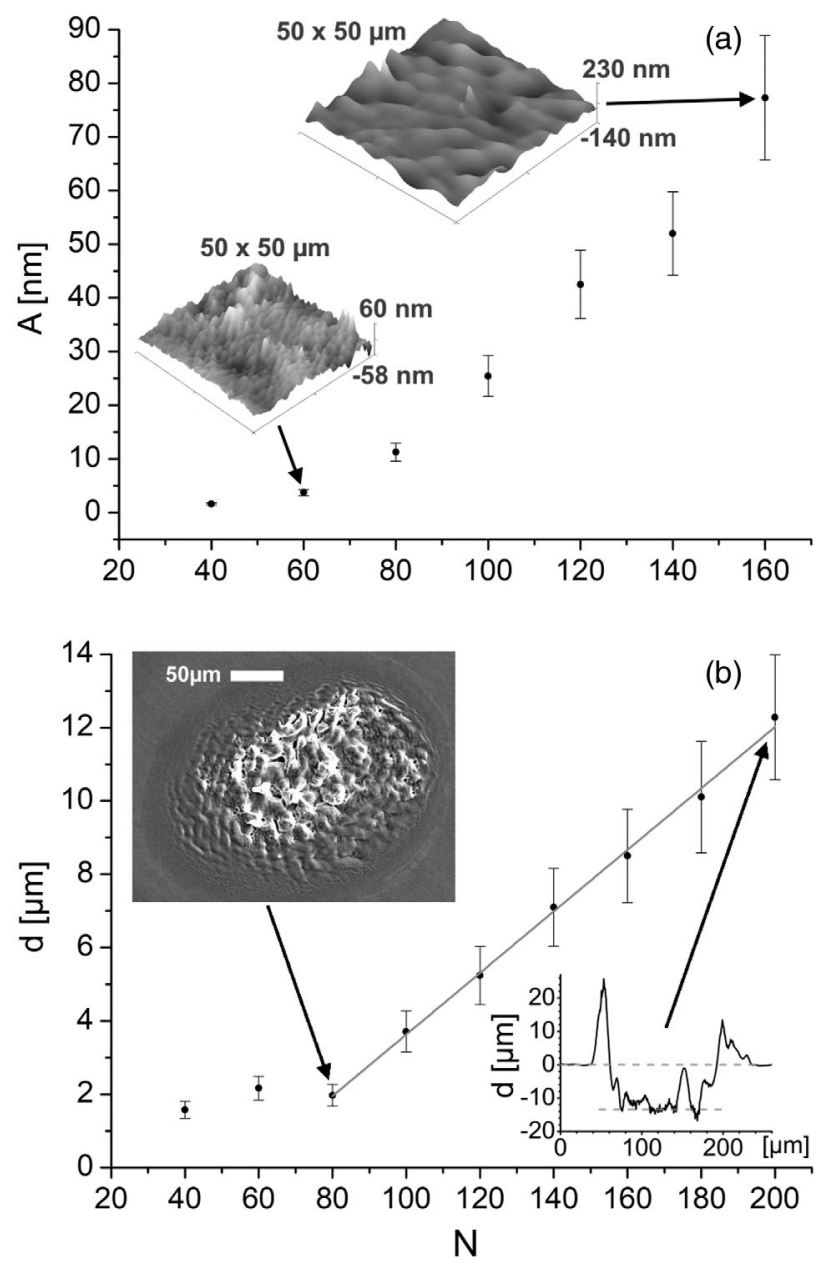

FIG. 4. (a) Amplitude of the dominating waves (averaged over ca. 20 different wave fronts), $A$, vs pulse number $N$ for a laser fluence of $0.68 \mathrm{~J} / \mathrm{cm}^{2}$ as determined from AFM micrographs. Two representative AFM micrographs $(50 \times 50 \mu \mathrm{m})$ corresponding to the data at two different points are presented in the insets in (a). (b) Ablation depth $d$ vs the pulse number for a laser fluence of $2.89 \mathrm{~J} / \mathrm{cm}^{2}$ as determined with OP. The insets in (b) show the shape of the crater as determined by SEM after 80 pulses and the crater profile after 200 pulses as determined by OP. The dashed lines emphasize the reference planes for the determination of the ablation depth.

fluence as shown in Fig. 3(b). Those features are dominating the morphology of the laser-irradiated $\mathrm{Nb}$ surfaces and contribute significantly to its surface shape and roughness. Their wavelength was found to be in the range of 2-13 $\mu \mathrm{m}$ for a weakly and a strongly ablated region, respectively. These values may be well related to the laser pulse duration [42]. Moreover, it is reasonable to assume that the observed wavelength of the surface profile depends on the interaction time of the laser pulse with the liquid metal and the vapor, leading to the formation of so-called laser-supported absorption waves [26]. They are related to pressure fluctuations of the expanding plasma over the target, which are acting on the liquefied surface. The maximum speed of the wave propagation for the liquid surface, in general, is the velocity of sound $v_{s}$. For liquid $\mathrm{Nb}$ close to the boiling point, $v_{s}$ is known to be well below $3889 \mathrm{~m} / \mathrm{s}$ [43]. Taking into account the measured wavelength $\lambda_{2}$ in Fig. 3(b), the interaction time of the laser beam with the plasma could be roughly estimated to have a maximum value of ca. $2.1 \mathrm{~ns}$ in the presented case, which is actually slightly smaller than the actual laser pulse duration of $3.5 \mathrm{~ns}$. Moreover, the wave amplitude $(A)$ in the melted region as determined from AFM micrographs increased for longer pulse trains as shown in Fig. 4(a), implying that the roughness of the melted layer grew as well. The power spectral density analysis of the AFM micrographs in comparison to the initial surface (see Fig. 5) confirms the formation of periodic structures on larger length scales in comparison to the initial surface, which is dominated by features on shorter length scales. The maximum wave amplitude detected prior to the occurrence of extensive boiling was found to be in the range of $350-400 \mathrm{~nm}$. This value corresponds well to the theoretically predicted maximum depth of the melted layer of ca. $700 \mathrm{~nm}$ [32].

The plot in Fig. 4(b) demonstrates a linear dependence of the etch depth (a distance between the initial surface and an averaged crater profile at the bottom) on the number of pulses as determined by OP, which could be observed only above a certain threshold in our experiment (e.g., $N=80$ for $F=2.89 \mathrm{~J} / \mathrm{cm}^{2}$ ) and is characteristic for the typical ablation regime [44-46], where an efficient removal or evaporation of surface material is expected to occur. The threshold of this ablation regime for $\mathrm{Nb}$ was previously reported to be around $5 \mathrm{~J} / \mathrm{cm}^{2}[47,48]$. Our slightly smaller value of the required laser fluence may be explained by the previously mentioned features; i.e. the laser pulses impinge on a surface with an increasingly altered shape, which is very likely to affect the melting behavior and the ablation threshold accordingly. Thus, we can conclude that the

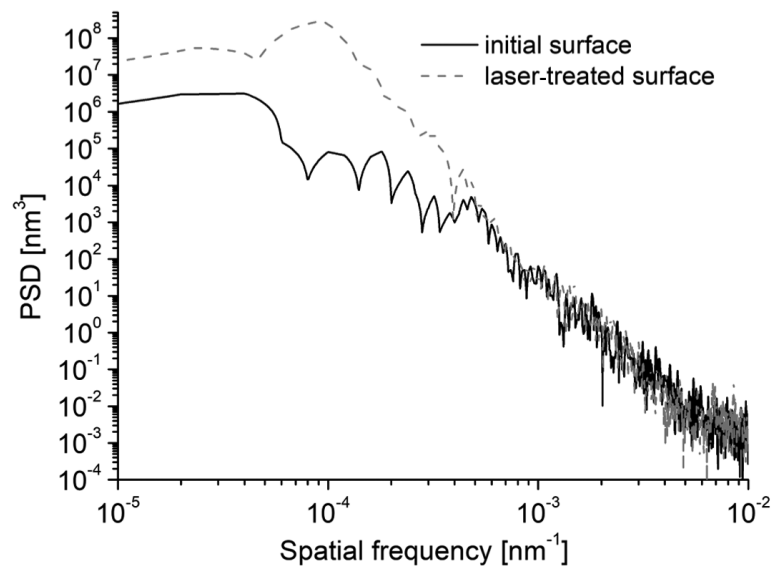

FIG. 5. (a) Power spectral density (PSD) of the initial surface in comparison to the laser-treated surface for $F=0.68 \mathrm{~J} / \mathrm{cm}^{2}$, $N=160$. The data correspond to the AFM micrograph $(50 \times 50 \mu \mathrm{m})$ presented in Fig. 4(a). 
ablation behavior is determined by the accumulation of the entire pretreatment of the sample surface.

As can be seen in the inset in Fig. 4(b), which depicts a SEM micrograph of the crater shape, the efficient ablation, in particular, results from an intense thermal evaporation of the liquid metal due to the boiling, the traces of which are clearly visible here. This kind of laser ablation led to the formation of pronounced crater edges and numerous defects distributed in the crater; see the SEM micrograph in the inset in Fig. 4(b), which presents the crater profile. In this context, it is important to note that a significant crater depth is already observed even for a smaller number of laser pulses, with no remarkable amounts of droplets spilled out. This observation confirms that it is sensible to already consider the presence of small pinholes as indicators for the presence of boiling.

Also, a small amount of droplets with a typical size in the range of 1-4 $\mu \mathrm{m}$ were splashed out of the craters; see Fig. 1(c). The molten material breaks down into vapor and droplets as a result of hydrodynamic instabilities, and a so-called "phase explosion" may occur; i.e. the overheated molten material becomes thermodynamically unstable approaching the critical point of the liquid-vapor system [49-52]. However, the applied laser fluence was well below the reported characteristic threshold of $15 \mathrm{~J} / \mathrm{cm}^{2}$ for $\mathrm{Nb}$ [47], and the multipulse processed craters exhibited numerous inhomogeneous rough structures such as cavities and protrusions. Thus, it is very likely that the critical temperature and pressure for the observed phase explosion could be reached only for individual protrusions of a sufficiently small size in our study.

\section{B. Field emission studies of laser-processed $\mathrm{Nb}$ surface}

After the laser processing, the $I_{\mathrm{FE}} E$ curves for all created spots were recorded in a voltage range of $0-10 \mathrm{kV}$ for working distances of 6-50 $\mu \mathrm{m}$ relative to the flat initial surface as the reference plane. The working distance was increased corresponding to higher pulse numbers and energy. In the case of melting without boiling, an average ablation depth relative to the initial surface increased slightly to values below $200 \mathrm{~nm}$. This additional distance was negligible relative to the working distance of the test electrode, and this effect is taken into account in the relative error. In the case of melting with boiling [see Fig. 4(b)], a significant material removal of several microns occurred, and erratic structures with a height of several microns were distributed randomly inside of the craters. These spots were measured with working distances of $50 \mu \mathrm{m}$ and evaluated relative to the reference plane. The field emission characteristics of the initial surface were investigated at ten random points for comparison. In general, the onset field $E_{\text {on }}$ was estimated for a local field emission current $I_{\mathrm{FE}}$ of $100 \mathrm{pA}$. The value of $E_{\text {on }}$ for the initial surface was found to be $(648 \pm 80) \mathrm{MV} / \mathrm{m}$. The $\beta$ values of the measured flat regions were expected to be at least very close to 1 .
However, an initial surface was in all cases activated due to an explosive breakdown. The main reason for the activation is an additional field enhancement in a thin, electrically isolating oxide layer as discussed in Ref. [53]. Moreover, the purity of this oxide layer and a subnanometer surface damage are essential factors, which involve the basic physics of an insulator breakdown here. The $I_{\mathrm{FE}} E$ behaviors as shown in Figs. 6(a)-6(c) are characteristic for the morphologies presented in Figs. 1(a)-1(c).

The surface with the above-described wavelike structures (see Fig. 3) and the surface with the small cavities [see Fig. 6(b)] exhibited rather high onset fields of ca. 717 [Fig. 6(a)] and ca. $618 \mathrm{MV} / \mathrm{m}$ [Fig. 6(b)], respectively, close to the value of the initial surface. In both cases, an erratic explosive activation of emitting structures resulted for the up cycle if a critical field is exceeded. Thus, reduced onset fields of 622 and $313 \mathrm{MV} / \mathrm{m}$ were observed for the down cycle in both cases. A subsequently conducted SEM analysis of the measured areas showed additional craters (see Fig. S4 [20]), which are characteristic for an explosive breakdown [54]. As presented in the insets in Figs. 6(a) and 6(b), additionally created surface irregularities emitted stable and confirmed the FN theory. The corresponding observed values of the field enhancement factors for the down cycle were found to be 6 and 12, respectively, and thus substantially larger than 1 .

The field emission threshold for the irregular deep craters [compare to Fig. 1(c)] as shown in Fig. 6(c) was measured to be around $72 \mathrm{MV} / \mathrm{m}$ only. An explosive breakdown was not observed in this case. Furthermore, the $I_{\mathrm{FE}} E$ curve showed an unstable behavior with a hysteresis and a shift of the onset field to lower values for the down cycle. A field enhancement factor of 17 was derived from the FN curve for the up cycle.

In total, 54 laser-processed spots on $\mathrm{Nb}(110)$ surfaces were investigated up to now. The distribution of the derived $\beta$ factors is presented in Fig. 7. An explosive breakdown occurred in ca. $50 \%$ of all measurements made so far. The $\beta$ values after the explosive activation were found to be smaller for the nonboiled areas and subsequently higher for the boiled areas. Furthermore, the $\beta$ values of the boiled regions ranged up to a value of 125 and were clearly substantially larger compared to the nonboiled, i.e. remelted, surface regions. This result can be expected due to the presence of numerous random structures with a height of several micrometers, in particular, inside and at the edges of the boiled craters.

In contrast, all nonboiled regions exhibited $\beta$ values smaller than 10 . Remarkably, these values are significantly lower compared to $\beta$ values of about $30-50$ that are typically observed for particles, scratches, and other surface irregularities $[55,56]$. The observed wavelike surface morphology was also used in numerical simulations (see Fig. S5 [20]), which predict $\beta$ values around 2 for our experimental conditions and assuming a wide range of 

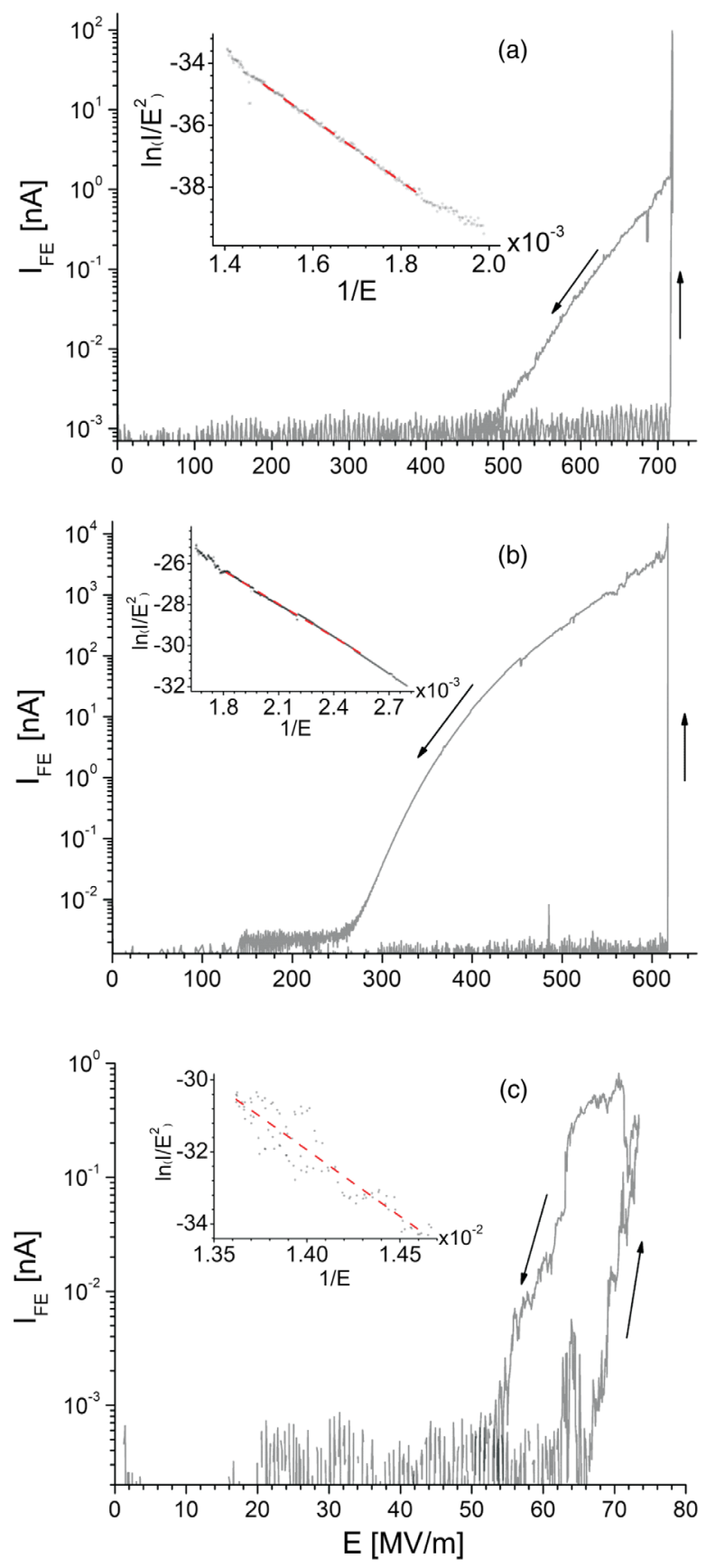

FIG. 6. Field emission current $I_{\mathrm{FE}}$ vs macroscopic electrical field $E$ for the craters created with the following laser parameters: $F=0.68 \mathrm{~J} / \mathrm{cm}^{2}, N=120$ (a); $F=1.40 \mathrm{~J} / \mathrm{cm}^{2}, N=100$ (b); and $F=2.89 \mathrm{~J} / \mathrm{cm}^{2}, N=140$ (c). The arrows emphasize the voltage cycle applied to the cathode. The insets show $I_{\mathrm{FE}} E$ curves as FN plots of the down cycle (a),(b) and of the up cycle (c).

periodic structures with different lateral wavelengths and vertical amplitudes as experimentally observed.

The onset field for the laser-processed spots in dependence of the number of pulses is presented in Fig. 8. These data directly correlate with the observed surface morphologies in Fig. 4. The surface melted with a laser fluence of $0.68 \mathrm{~J} / \mathrm{cm}^{2}$ [see Fig. 8(a)] exhibited a high onset field

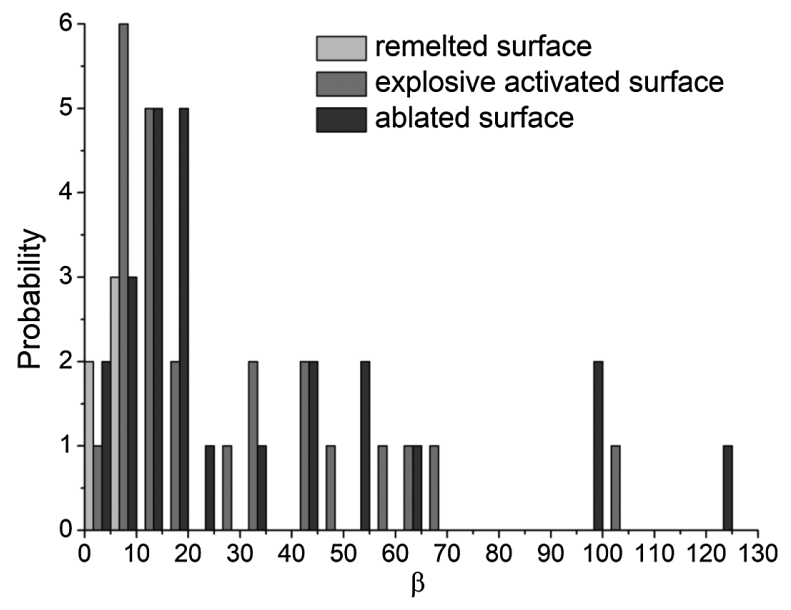

FIG. 7. Distribution of the field enhancement factors, $\beta$, derived from the $I_{\mathrm{FE}} E$ curves for boiled craters, slightly melted regions, and after the explosive breakdowns.

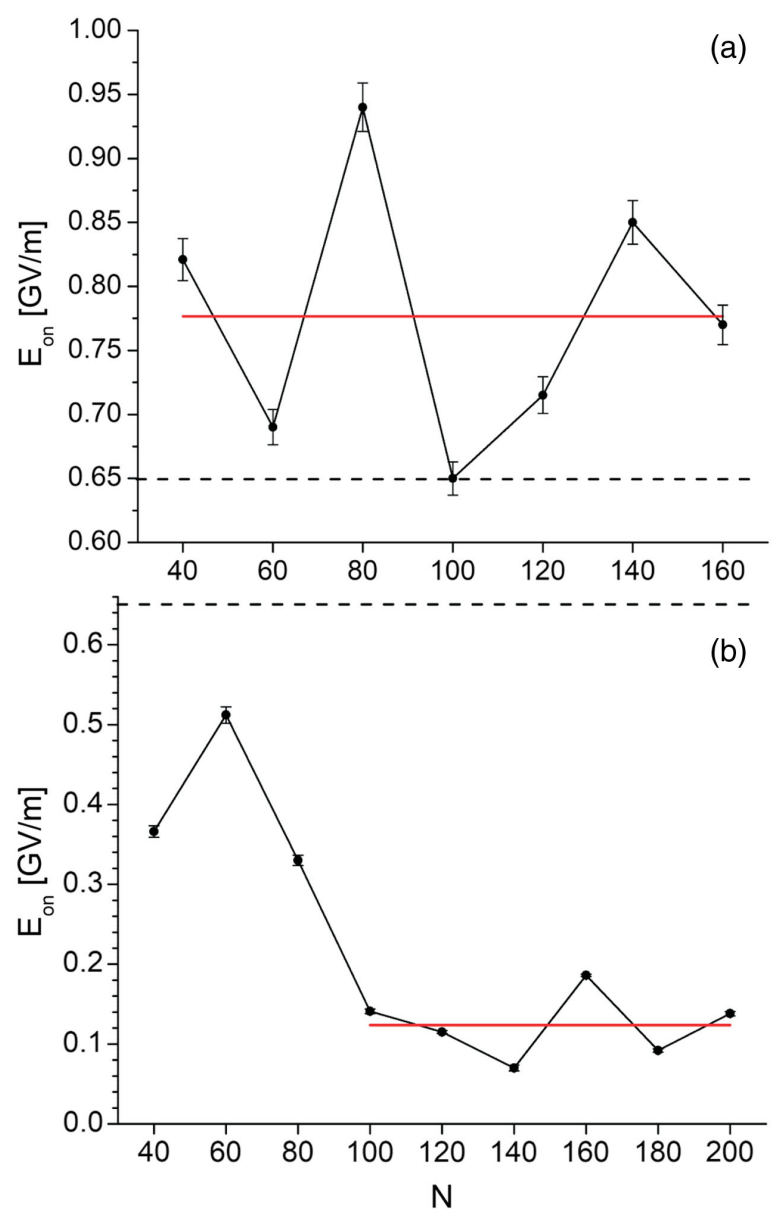

FIG. 8. Field emission onset field $E_{\mathrm{on}}$, measured by $I_{\mathrm{FE}}=$ $100 \mathrm{pA}$ vs pulse number $N$, for the laser-processed surface with laser fluences of 0.68 (a) and $2.89 \mathrm{~J} / \mathrm{cm}^{2}$ (b), respectively. The solid line emphasizes an average value for the entire range of data in (a) and the onset field data obtained for more than 100 pulses in (b). The dashed lines show $E_{\text {on }}$ for the initial surfaces for comparison. 
of $(776 \pm 38) \mathrm{MV} / \mathrm{m}$ independent on the magnitude of the wave structures; e.g., waves with an amplitude of up to $80 \mathrm{~nm}$ were measured for $N=160$. Thus, a higher surface roughness in this case did not shift the field emission threshold to lower values of the electric field, contrarily to the expectation. The onset field appeared to be rather high in comparison to the initial value. In the case of the illumination with a variable number of pulses while applying a laser fluence of $2.89 \mathrm{~J} / \mathrm{cm}^{2}$ [see Fig. 8(b)], the onset field clearly decreased with an increasing ablation depth for values of $N$ above 100. In the latter case, a significant removal of material from the illuminated regions has occurred. Hence, the final field emission threshold typically measured at the craters, which were deeper than $2 \mu \mathrm{m}$, could be estimated to $(124 \pm 17) \mathrm{MV} / \mathrm{m}$ only. It should be noted that the same behavior was observed also for $F=2.07 \mathrm{~J} / \mathrm{cm}^{2}, F=3.53 \mathrm{~J} / \mathrm{cm}^{2}$, and $F=4.27 \mathrm{~J} / \mathrm{cm}^{2}$. Overall, a rather high onset field of the nonboiled laser-processed area may be explained by the low field enhancement of the smoothed wavelike structures discussed above and by an improvement of the surface crystallinity due to an epitaxial regrowth of the upper layers (see Ref. [37]).

\section{CONCLUSIONS}

Surface modifications of flat $\mathrm{Nb}$ (110) single crystals due to nanosecond-pulsed laser illumination were studied in UHV at $4 \times 10^{-6} \mathrm{~Pa}$. The surface could be slightly melted for laser fluences of around $0.68 \mathrm{~J} / \mathrm{cm}^{2}$ showing different wave structures, while a substantial melting occurs for a higher laser fluence and an increasing number of pulses introducing boiling traces as well. Periodically corrugated structures of two different types systematically appeared in the remelted region on two different length scales. Waves with rather small amplitudes of below $20 \mathrm{~nm}$ and a lateral wavelength of about $355 \mathrm{~nm}$ induced directly during the laser illumination dominated in the boundaries of the illuminated areas. Waves with larger amplitudes of several hundred nanometers and a wavelength in the micron range propagated in the center part of the irradiated spot. A significant material removal was observed to occur by applying laser energies well below the expected ablation threshold of $5 \mathrm{~J} / \mathrm{cm}^{2}$ for multipulse excitations with a rather low repetition rate of $10 \mathrm{~Hz}$.

Field emission measurements of the laser-processed areas show a correlation between morphological surface changes, field enhancement values, and onset fields. The $\beta$ values after an explosive breakdown are considered in the study as well. Boiled craters exhibit rather low onset fields about $(124 \pm 17) \mathrm{MV} / \mathrm{m}$ and a higher field enhancement compared to slightly remelted regions. A high onset field of $(776 \pm 38) \mathrm{MV} / \mathrm{m}$ and $\beta$ values below 10 were found to be characteristic for the area remelted with $0.68 \mathrm{~J} / \mathrm{cm}^{2}$, despite an increased surface roughness due to numerous wavelike structures with amplitudes around $80 \mathrm{~nm}$. Furthermore, it can be concluded that a surface smoothed by a suited laser illumination can resist high electrical fields close to the theoretical threshold for field emission of around $1 \mathrm{GV} / \mathrm{m}$. Thus, a parasitic field emission of SRF cavities can be significantly suppressed and higher acceleration gradients can be achieved even if moderate ripple patterns induced by the laser illumination are present. Taking into account this fact, an actual laser treatment thus not requires a high laser energy stability, which is also an important factor for a practical application. In addition, a comparison of a simulated field enhancement factors with the measured ones revealed that the field emission behavior of a laser-processed surface can be better numerically predicted as in the case of the initial surface with random defects after a chemical polishing.

\section{ACKNOWLEDGMENTS}

The research was funded by the German Federal Ministry of Education and Research under Project No. 05H15PXRB1.

[1] E. Ukar, A. Lamikiz, L. N. Lopez de Lacalle, D. del Pozo, and J. L. Arana, Laser polishing of tool steel with $\mathrm{CO}_{2}$ laser and high-power diode laser, International Journal of machine tools and manufacture 50, 115 (2010).

[2] L. Giorleo, E. Ceretti, and C. Giardini, Ti surface laser polishing: Effect of laser path and assist gas, Proc. CIRP 33, 446 (2015).

[3] C. D. Giorgi, V. Furlan, A. G. Demir, E. Tallarita, G. Candiani, and B. Previtali, Laser micro-polishing of stainless steel for antibacterial surface applications, Proc. CIRP 49, 88 (2016).

[4] K. M. Nowak, H. J. Baker, and D. R. Hall, Efficient laser polishing of silica micro-optic components, Appl. Opt. 45, 162 (2006).

[5] J. Schmidt, R. Scholz, and H. Riegel, Laser polishing of aluminum by remelting with high energy pulses, Materialwiss. Werkstofftech. 46, 686 (2015).

[6] C. P. Ma, Y.C. Guan, and W. Zhou, Laser polishing of additive manufactured Ti alloys, Opt. Lasers Eng. 93, 171 (2017).

[7] B. N. Chicbkov, C. Momma, S. Nolte, F. von Alvensleben, and A. Tünnermann, Femtosecond, picosecond and nanosecond laser ablation of solids, Appl. Phys. A 63, 109 (1996).

[8] C. Nüsser, I. Wehrmann, and E. Willenborg, Influence of intensity distribution and pulse duration on laser micro polishing, Phys. Procedia 12, 462 (2011).

[9] E. V. Bordatchev, A. M. K. Hafiz, and O. R. Tutunea-Fatan, Performance of laser polishing in finishing of metallic surfaces, International Journal of Advanced Manufacturing Technology 73, 35 (2014).

[10] B. Rosa, P. Mognol, and J. Hascoët, Laser polishing of additive laser manufacturing surfaces, J. Laser Appl. 27, S29102 (2015). 
[11] V. Palmieri, F. Stivanello, S. Yu. Stark, C. Roncolato, and M. Valentino, Besides the standard niobium bath chemical polishing, in Proceedings of the 10th Workshop on RF Superconductivity (KEK, Tsukuba, 2001), p. 408, https:// accelconf.web.cern.ch/accelconf/srf01/papers/pr016.pdf.

[12] W. Singer, X. Singer, A. Brinkmann, J. Iversen, A. Matheisen, A. Navitski, Y. Tamashevich, P. Michelato, and L. Monaco, Superconducting cavity material for the European XFEL, Supercond. Sci. Technol. 28, 085014 (2015).

[13] D. Moffat, P. Barnes, T. Flynn, J. Graber, L. Hand, W. Hartung, T. Hayes, J. Kirchgessner, J. Knobloch, R. Noer, H. Padamsee, D. Rubin, and J. Sears, Studies on the nature of field emission sites, in Proceedings of the 5th Workshop on RF Superconductivity (DESY, Hamburg, 1991), p. 245, https://accelconf.web.cern.ch/accelconf/SRF91/papers/ srf91d02.pdf.

[14] J. Knobloch, R. L. Geng, M. Liepe, and H. Padamsee, High-field Q slope in superconducting cavities due to magnetic field enhancement at grain boundaries, in Proceedings of the 9th Workshop on RF Superconductivity (LANL, Los Alamos, 1999), p. 77, https://laacg.lanl.gov/ rfsc99/rfsc99_web/TUA/tua004.pdf.

[15] V. Shemelin and H. Padamsee, Magnetic field enhancement at pits and bumps on the surface of superconducting cavities, DESY, Report No. TTC-Report 2008-07, 2008, http://citeseerx.ist.psu.edu/viewdoc/download?doi=10.1.1 $.504 .9256 \&$ rep $;=$ rep $1 \&$ type $;=$ pdf.

[16] A. Navitski, S. Lagotzky, D. Reschke, X. Singer, and G. Müller, Field emitter activation on cleaned crystalline niobium surfaces relevant for superconducting rf technology, Phys. Rev. ST Accel. Beams 16, 112001 (2013).

[17] A. Aspart and C.Z. Antoine, Study of the chemical behavior of hydrofluoric, nitric and sulfuric acids mixtures applied to niobium polishing, Appl. Surf. Sci. 227, 17 (2004).

[18] L. Zhao, J. M. Klopf, C. E. Reece, and M. J. Kelley, Laser polishing of niobium for superconducting radio-frequency accelerator applications, Phys. Rev. ST Accel. Beams 17, 083502 (2014).

[19] P. Kneisel and B. Lewis, Advanced surface cleaning methods-three years of experience with high pressure ultrapure water rinsing of superconducting cavities, in Proceedings of the 7th Workshop on RF Superconductivity (CEA/Saclay, Gif-sur-Yvette, 1995), p. 311, http:// accelconf.web.cern.ch/Accelconf/SRF95/papers/srf95102 .pdf.

[20] See Supplemental Material at http://link.aps.org/ supplemental/10.1103/PhysRevAccelBeams.22.023101 for more detailed information on the experimental setup and its electrical characteristics. Furthermore, a picture illustrating the setup for the field emission measurements as well as a SEM micrograph of a sample after an explosive activation are given.

[21] I. H. Malitson, Interspecimen comparison of the refractive index of fused silica, J. Opt. Soc. Am. 55, 1205 (1965).

[22] D. Lysenkov and G. Müller, Field emission measurement techniques for the optimization of carbon nanotube cathodes, Int. J. Nanotechnology 2, 239 (2005).
[23] R. G. Forbes, Field emission: New theory for the derivation of emission area from a Fowler-Nordheim plot, J. Vac. Sci. Technol. B 17, 526 (1999).

[24] B. J. Hopkins and M. Ibrahim, Oxygen adsorption on the (110) face of tantalum, niobium, molybdenum and tungsten single crystals, Vacuum 23, 135 (1973).

[25] J. H. Weaver, D. W. Lynch, and C. G. Olson, Optical properties of niobium from 0.1 to $36.4 \mathrm{eV}$, Phys. Rev. B 7, 4311 (1973).

[26] P. Schaaf, Laser Processing of Materials (Springer, Berlin, 2010), pp. 28-44.

[27] S. Amoruso, Modeling of UV pulsed-laser ablation of metallic targets, Appl. Phys. A 69, 323 (1999).

[28] N. Farid, S. S. Harilal, H. Ding, and A. Hassanein, Emission features and expansion dynamics of nanosecond laser ablation plumes at different ambient pressures, J. Appl. Phys. 115, 033107 (2014).

[29] C. Sawyer, K. Iyer, X. Zhu, and M. K. Luke, and D. Amdahl, Two-dimensional laser-induced thermal ablation modeling with integrated melt flow and vapor dynamics, J. Laser Appl. 29, 022212 (2017).

[30] J. Reif, F. Costache, and M. Bestehorn, in Recent Advances in Laser Processing of Materials, edited by J. Perriere, E. Millon, and E. Fogarassy (Elsevier Science, New York, 2006), p. 275.

[31] V. N. Tokarev, J. G. Lunney, W. Marine, and M. Sentis, Analytical thermal model of ultraviolet laser ablation with single-photon absorption in the plume, J. Appl. Phys. 78, 1241 (1995).

[32] N. M. Bulgakova, A. V. Bulgakov, and L. P. Babich, Energy balance of pulsed laser ablation: Thermal model revised, Appl. Phys. A 79, 1323 (2004).

[33] S. Singaravelu, J. M. Klopf, G. Krafft, and M. J. Kelley, Laser nitriding of niobium for application to superconducting radio-frequency accelerator cavities, J. Vac. Sci. Technol. B 29, 061803 (2011).

[34] S. Sinha, Thermal model for nanosecond laser ablation of alumina, Ceram. Int. 41, 6596 (2015).

[35] I. V. Guk, G. D. Shandybina, and E. B. Yakovlev, Role of the heat accumulation effect in the multipulse modes of the femtosecond laser microstructuring of silicon, Semiconductors 50, 694 (2016).

[36] Y. Lee, M. F. Becker, and R. M. Walser, Laser-induced damage on single-crystal metal surfaces, J. Opt. Soc. Am. B 5, 648 (1988).

[37] L. Buene, J. M. Poate, D. C. Jacobson, C. W. Draper, and J. K. Hirvonen, Laser irradiation of nickel single crystals, Appl. Phys. Lett. 37, 385 (1980).

[38] O. Armbruster, A. Naghilou, M. Kitzler, and W. Kautek, Spot size and pulse number dependence of femtosecond laser ablation thresholds of silicon and stainless steel, Appl. Surf. Sci. 396, 1736 (2017).

[39] M. Bass and L. Liou, Calorimetric studies of light absorption by diamond turned $\mathrm{Ag}$ and $\mathrm{Cu}$ surfaces and analyses including surface roughness contributions, J. Appl. Phys. 56, 184 (1984).

[40] J. E. Sipe, J. F. Young, J. S. Preston, and H. M. van Driel, Laser-induced periodic surface structure. I. Theory, Phys. Rev. B 27, 1141 (1983). 
[41] M. V. Shugaev, I. Gnilitskyi, N. M. Bulgakova, and L. V. Zhigilei, Mechanism of single-pulse ablative generation of laser-induced periodic surface structures, Phys. Rev. B 96, 205429 (2017).

[42] M. S. Trtica, B. M. Gakovic, B. B. Radak, D. Batani, T. Desai, and M. Bussoli, Periodic surface structures on crystalline silicon created by $532 \mathrm{~nm}$ picosecond $\mathrm{Nd}$ : YAG laser pulses, Appl. Surf. Sci. 254, 1377 (2007).

[43] R.S. Hixson and M. A. Winkler, Thermophysical properties of liquid niobium, High Press. Res. 4, 555 (1990).

[44] R. D. Vispute, S. Choopun, R. Enck, A. Patel, V. Talyansky, R. P. Sharma, T. Venkatesan, W. L. Sarney, L. Salamancariba, S. N. Andronescu, A. A. Iliadis, and K. A. Jones, Characterization of phosphorus implantation in $4 \mathrm{H}-\mathrm{SiC}$, J. Electron. Mater. 28, 275 (1999).

[45] M. E Shaheen, J. E Gagnon, and B. J Fryer, Excimer laser ablation of aluminum: Influence of spot size on ablation rate, Laser Phys. 26, 116102 (2016).

[46] A. Miotello and P. M. Ossi, Laser-Surface Interactions for New Materials Production (Springer, Berlin, 2010), p. 152.

[47] N. M. Bulgakova and A. V. Bulgakov, Pulsed laser ablation of solids: Transition from normal vaporization to phase explosion, Appl. Phys. A 73, 199 (2001).

[48] N. A. S. Rodrigues, M. A. P Gião, C. A. B. Silveira, R. Riva, and C. Schwab, Ablation of molybdenum and niobium with a HyBrID copper laser, Appl. Surf. Sci. 200, 68 (2002).
[49] J. H. Yoo, S. H. Jeong, X. L. Mao, R. Greif, and R. E. Russo, Evidence for phase-explosion and generation of large particles during high power nanosecond laser ablation of silicon, Appl. Phys. Lett. 76, 783 (2000).

[50] L. V. Zhigilei, Dynamics of the plume formation and parameters of the ejected clusters in short-pulse laser ablation, Appl. Phys. A 76, 339 (2003).

[51] L. V. Zhigilei, Z. Lin, and D. S. Ivanov, Atomistic modeling of short pulse laser ablation of metals: Connections between melting, spallation, and phase explosion, J. Phys. Chem. C 113, 11892 (2009).

[52] C. Porneala and D. A. Willis, Time-resolved dynamics of nanosecond laser-induced phase explosion, J. Phys. D 42, 155503 (2009).

[53] S. Lagotzky and G. Müller, Statistical model for field emitter activation on metallic surfaces used in highgradient accelerating structures, Nucl. Instrum. Methods Phys. Res., Sect. A 806, 193 (2016).

[54] G. A. Mesyats, Ectons and their role in plasma processes, Plasma Phys. Controlled Fusion 47, A109 (2005).

[55] J. Noer, P. Niedermann, N. Sankarraman, and O. Fischer, Electron field emission from intentionally introduced particles on extended niobium surfaces, J. Appl. Phys. 59, 3851 (1986).

[56] A. D. Pandey, G. Müller, D. Reschke, and X. Singer, Field emission from crystalline niobium, Phys. Rev. ST Accel. Beams 12, 023501 (2009). 